\title{
Interpretação geométrica dos signos das razões trigonométricas com Geogebra
}

Geometric interpretation of the signs of trigonometric ratios with Geogebra

\author{
Stephanie Chiquinquira Diaz Urdaneta ${ }^{1}$ \\ Juan Luis Prieto Gonzalez ${ }^{2}$ \\ Ana Duarte Castillo ${ }^{3}$
}

\section{Resumo}

O ensino da Trigonométrica caracterizou-se por um enfoque algébrico, o que tem feito de seu estudo um processo memorístico e mecânico. Um conteúdo onde se pode evidenciar o anterior é o dos signos das razões trigonométricas que costuma se abordar através do uso de regras mnemotécnicas, ação que limita seu entendimento. Com a intenção de apoiar à superação deste problema, neste trabalho descreve-se o desenho de um recurso elaborado com o software GeoGebra e sua aplicação em uma sequência para analisar e dotar sentido aos signos das raciocine Seno, Cosseno e Tangente de um ângulo. $O$ desenho do recurso baseou-se no modelo TPACK (Mishra \& Koehler, 2006). Se considera apropriado que os professores contem com recursos deste estilo que lhe ajudem em suas práticas de ensino apoiadas em meios tecnológicos como o GeoGebra.

Palavras chave: razões trigonométricas; regras mnemotécnicas; GeoGebra

\begin{abstract}
The teaching of Trigonometry has been characterized by an algebraic approach, which has made its study a memoristic and a mechanical process. A content where the previous can be evidenced is the signs of the trigonometric ratios, which tend to be treated through the use of mnemonic devices, action that limits its comprehension. With the aim of supporting the overcoming of this problem, in this paper it is described the design of an elaborated resource with the software GeoGebra and its application in a sequence to analyze and make sense of the Sine, Cosine and Tangent ratios. The design of the resource was based on the TPACK model (Mishra \& Koehler, 2006). It is deemed pertinent that teachers have this style of resources in the sense that these help them in their teaching practices supported on technological environments like GeoGebra.
\end{abstract}

Keywords: trigonometric ratios, mnemonic devices, GeoGebra

\footnotetext{
1 Asociación Aprender en Red. Grupo TEM: Tecnologías en la Educación Matemática - Venezuela | stephaniediazurdaneta@gmail.com

2 Universidad del Zulia. Asociación Aprender en Red. Grupo TEM: Tecnologías en la Educación Matemática Venezuela | juanl.prietog@gmail.com

3 Universidade Federal do Pará | aduarte@una.edu.ve
} 


\section{Introdução}

Certas investigações assinalam que quanto mais representações de um objeto matemático se tenham, poderá existir um melhor entendimento do mesmo (BORBA, 1993; BORBA \& VILLAREAL, 2005; DUVAL, 1996; GUTIÉRREZ \& PRIETO, 2015). No entanto, o estudo da trigonometria caracterizou-se por um enfoque mais algébrico que limita 0 entendimento de seus conceitos e propriedades, e não permite estabelecer relações entre suas diferentes representações (FIALLO, 2010).

Alguns autores assinalam certas razões que podem ser considerado como consequências do uso do referido enfoque: (i) as deficiências no manejo de certos conteúdos trigonométricos por parte dos professores (BRITO \& MOREY, 2004; CHACÓN, SÁNCHEZ \& QUIRÓS, 2007; FI, 2006) e (ii) a abordagem tradicionalista dos textos escolares (Fiallo, 2010). Um ensino da trigonometria sob estas condições gera no estudante certas dificuldades para compreender esta área da Matemática, tendo como consequência a conversão dos estudos em um processo memorístico, rotineiro e mecânico (FIALLO e GUTIÉRREZ, 2007).

Um dos conteúdos onde se pode evidenciar esta problemática são os signos das razões trigonométricas, tópico que os professores costumam tratar por meio do uso de regras práticas ou mnemotécnicas (FIALLO, 2010; DÍAZ e PRIETO, 2013) (Figura 1). Tal ação, por parte do professor, tem como consequência que os estudantes se vejam forçados a memorizar o signo da cada razão trigonométrica para a cada um dos quatro quadrantes do plano cartesiano, sem sentido algum. Diante desta realidade, considera-se importante a abordagem dos signos das razões trigonométricas através de sua interpretação geométrica desde a circunferência unitária, meio que se considera mais apropriado, já que permite a visualização de ditos signos através de sua representação a partir de algum objeto geométrico que permita-o caracterizar.

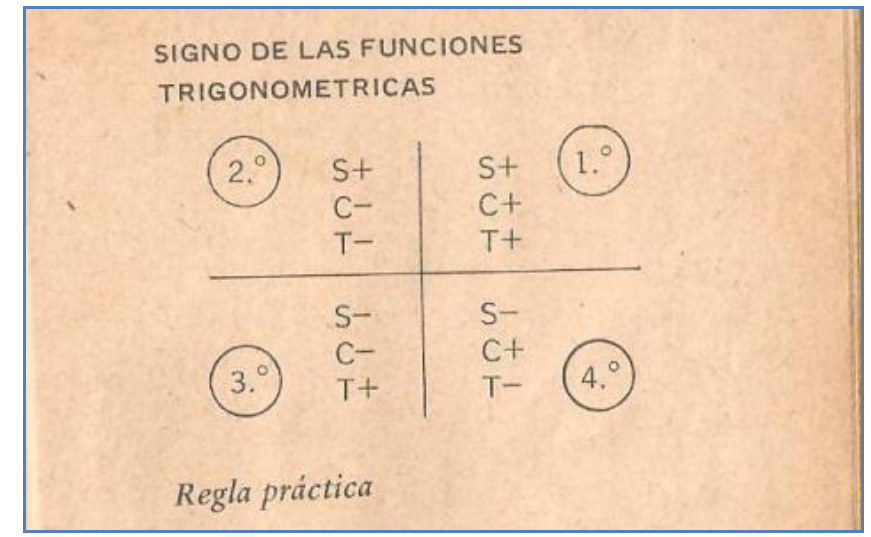

Figura 1. Signos das razões trigonométricas. (NAVARRO, 1970)

Atualmente, a abordagem de conteúdos matemáticos que requerem de interpretação geométrica mediante o uso de recursos tecnológicos tem trazido benefícios e melhoras na aprendizagem dos estudantes (LU, 2008). Isso se deve especialmente às diversas atividades que são promovidas através destes meios e à qualidade dos recursos desenhados. (LABORDE, KYNIGOS, HOLLEBRANDS E STRÄSSER, 2006). Vincula-se a qualidade de um recurso desenhado e ao uso de uma sequência que os professores podem levar a cabo durante o desenvolvimento do tema. Então, é preciso garantir que o estudante obtenha condições favoráveis que the permitam dotar de sentido os signos das regras 
mnemotécnicas, de maneira que possa transcender o uso destas regras, compreendendoas, aos mesmo tempo que as memoriza.

Entre os recursos tecnológicos que têm beneficiado a aprendizagem dos estudantes, selecionou-se o GeoGebra, por se tratar de um tipo especial de Software de Geometria Dinâmica (SGD), de acesso livre e de código aberto, que combina, em tempo real, as representações gráficas e expressões simbólicas de diversos objetos matemáticos (DIKOVIĆ, 2009; HOHENWARTER, 2006).

Nesse sentido, este trabalho descreve o desenho de um recurso elaborado com o GeoGebra e sua aplicação em uma sequência didática para analisar e dotar sentido os signos que representam o Seno, Cosseno e Tangente de um ângulo. Isso nos permitiu estudar o signo das razões trigonométricas na circunferência unitária observando o comportamento de um objeto geométrico sócio à cada uma das razões e com isso compreender ditos signos através de sua interpretação geométrica e confirmar, de forma visual, os resultados estabelecidos nas regras mnemotécnicas.

\section{O desenho do recurso}

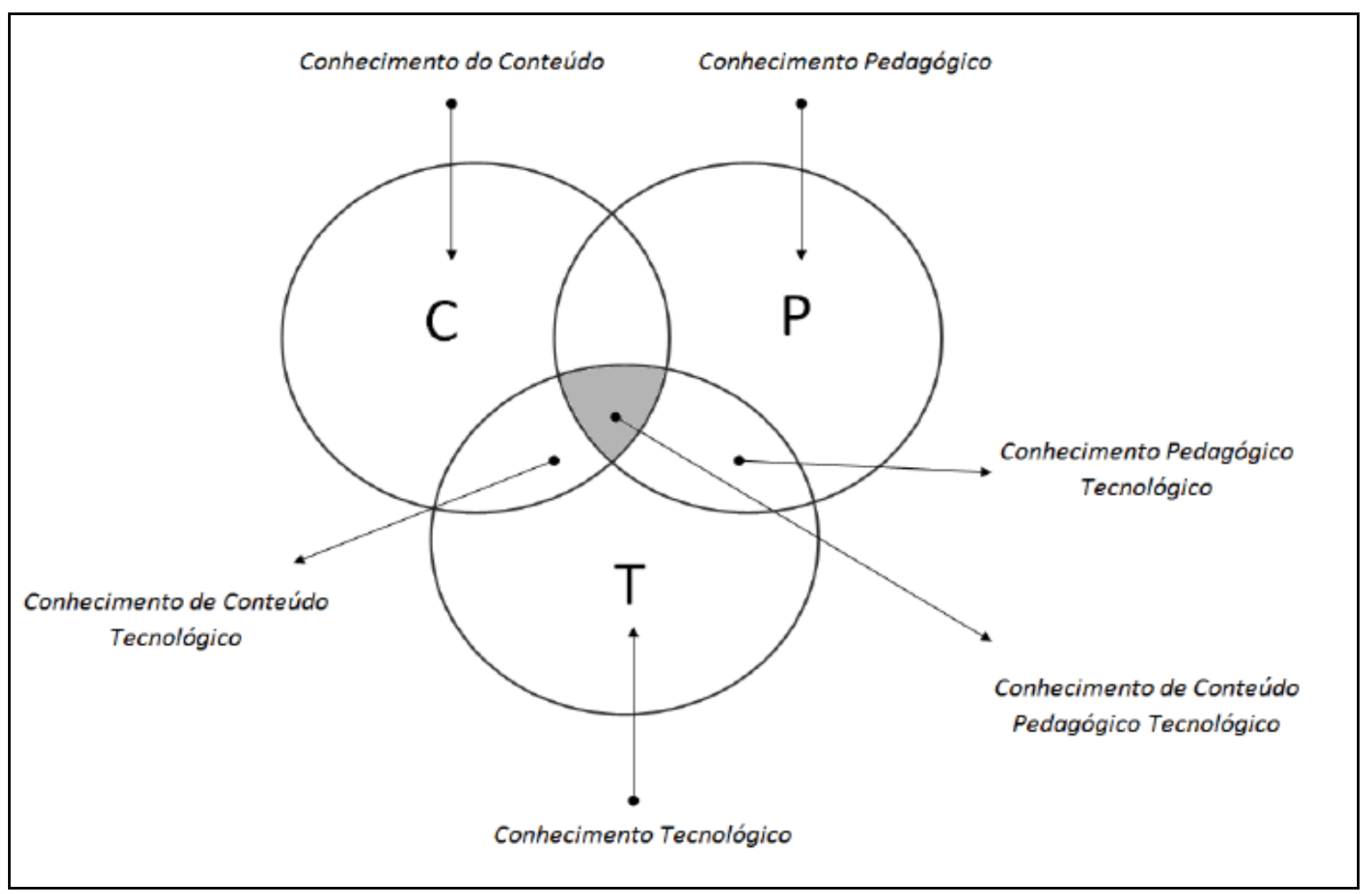

Figura 2. Modelo TPACK (adaptado de MISHRA \& KOEHLER, 2006, p. 1025)

Para o desenho do recurso aqui descrito, utilizou-se o Software de Geometria Dinâmica GeoGebra como meio para definir uma sequência de ensino do conteúdo dos signos das razões trigonométricas Seno, Cosseno e Tangente de um ângulo, sob o contexto da circunferência unitária. O desenho da sequência de ensino estruturou-se levando em conta a natureza do conhecimento que se manifesta por parte do professor de Matemática ao realizar tarefas deste tipo. Neste caso, o modelo utilizado foi o TPACK, definida por Mishra \& Koehler (2006) como uma forma emergente de conhecimento, pois vai além do conteúdo 
em si, da pedagogia como princípio e da tecnologia digital disponível, situa-se nas interações entre estes conhecimentos.

Agora bem, a figura 2, adaptada de Mishra \& Koelher (2006) representa graficamente o conceito de TPACK como sendo o resultado da intersecção do conhecimento de um professor em três níveis: conhecimento dos conteúdos curriculares, dos métodos pedagógicos e ainda as competências a nível tecnológico.

Segundo Coutinho (2011), na opinião de Koehler \& Mishra (2008), o TPACK é a base de um ensino eficaz com as tecnologias e condição para uma eficiente inserção das TICs nas atividades curriculares. Seu domínio exige uma compreensão por parte do professor das técnicas pedagógicas que possibilitam que as tecnologias sejam usadas em prol da construção do saber pelo aluno e não como um apoio ao professor para ensinar.

Nesse sentido, e segundo os mesmos autores, a formação de professores deve ser direcionada para o desenvolvimento do TPACK numa forma gradual e em espiral, começando a formação com as tecnologias mais simples e que os professores já conhecem, rumo a aplicações cada vez mais complexas e sofisticadas. No fundo, o que se pretende, é que o professor seja capaz de tomar decisões fundamentadas no desenho das suas atividades de ensino com as tecnologias o que, segundo Cox (2008) pressupõe: a) saber usar as tecnologias; b) numa dada área curricular, c) integrada numa estratégia pedagógica especifica d) num determinado contexto educativo, e) para promover a construção do conhecimento do aluno, relativo a um determinado conteúdo/tópico programático e/ou para contribuir para a consecução de um objetivo educacional previamente identificado.

Quanto ao desenho da sequência de ensino, o conhecimento por parte do professor de Matemática ao realizar tarefas deste tipo, se apresenta em forma de considerações conceituais, the proporcionando um instrumental didático que será descrito a seguir.

\section{Considerações Conceituais}

Devido à particularidade deste conteúdo, foi necessário definir os seguintes objetos matemáticos envolvidos na análise:

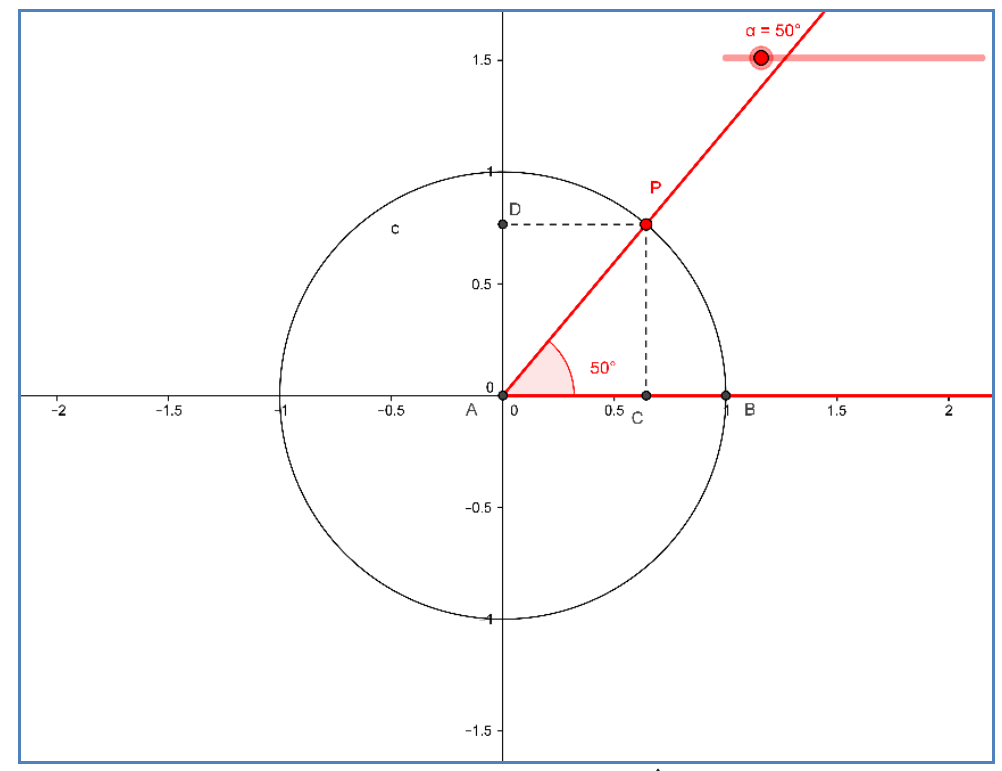

Figura 3. Circunferencia Unitária e Ângulo Central 
Circunferência unitária: também conhecida como circunferência trigonométrica (ou círculo trigonométrico), tem como centro na origem do sistema de coordenadas cartesianas e sua rádio tanto faz à unidade. Na sequência, esta circunferência é considerada o médio para a interpretação geométrica do signo de raciocine-as Seno, Cosseno e Tangente (Ver Figura 3).

Ângulo central. sobre a circunferência desenha-se um ângulo central com vértice no centro da mesma, onde um de seus lados está fixo na parte positiva do e o outro lado ocupa qualquer posição no plano, segundo seja a amplitude do ângulo. Este último lado do ângulo corta a circunferência trigonométrica em um ponto, a partir do qual se traçam segmentos perpendiculares até os eixos coordenados. Os triângulos retângulos que se formam a partir desta construção também são parte da análise (ver Figura 3).

É importante destacar que outras construções auxiliares, explicadas mais adiante, permitirão representar outros triângulos retângulos que resultam mais úteis para realizar o estudo.

Razões trigonométricas. a partir dos triângulos retângulos formados na circunferência unitária, desde uma perspectiva geométrica, é possível definir estas razões da seguinte maneira:

Seno do ângulo $\alpha$ (abreviado Sen $\alpha$ ) está determinado pela razão $\frac{\overline{P C}}{\overline{A P}}$ entre o cateto oposto ao ângulo $\overline{P C}$ e a hipotenusa $\overline{A P}$ do triângulo $A P C$ da figura 1. Desde um ponto de vista geométrico, dado que $\overline{A P}=1$ é constante, o seno $\alpha$ fica referido pelo segmento $P C$. Para a análise tomou-se a projeção ortogonal deste segmento sobre o eixo $y_{\text {, o segmento }}$ $\overline{A D}$, este se considerou um segmento dirigido, isto é o vetor $\overrightarrow{A D}$. Desta maneira o Sen $\alpha$ fica representado pelo módulo e sentido do vetor posição $\overrightarrow{A D}$ de cor azul (ver Figura 4 a).

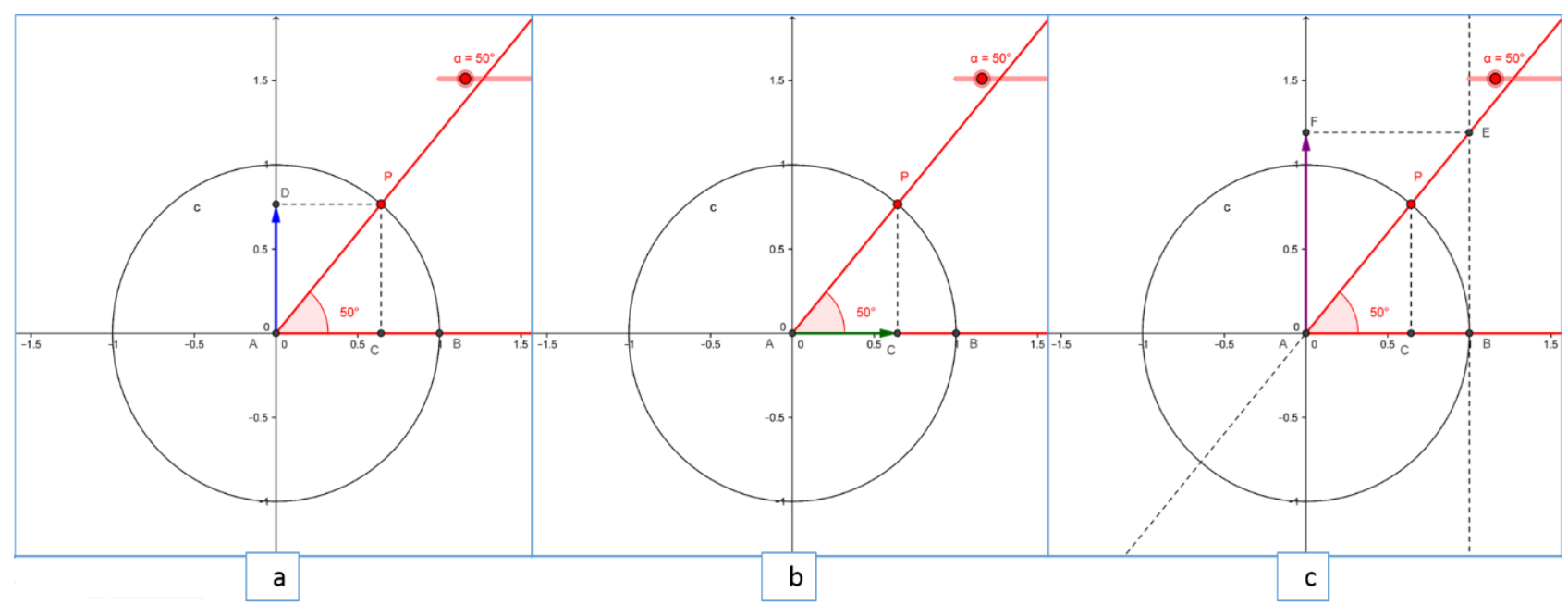

Figura 4. Razões Seno, Cosseno e Tangente.

Cosseno do ângulo $\alpha$ (abreviado $\operatorname{Cos} \alpha$ ) está determinado pela razão $\frac{\overline{A C}}{\overline{A P}}$ entre o cateto adjacente ao ângulo $\overline{A C}$ e a hipotenusa $\overline{A P}$ do triângulo $A P C$. Dado que $A P=1,0$ referente ao $\operatorname{Cos} \alpha$ é o segmento $\overline{A C}$ considerou-se como um segmento dirigido, o vetor $\overrightarrow{A C}$. Para este caso, dito vetor é posição e está contido no eixo $x$ (ver Figura 4b). Dado o anterior, o $\operatorname{Cos} \alpha$ fica representado pelo sentido e módulo do vetor posição $\overrightarrow{A C}$ de cor verde. 
A Tangente do ângulo $\alpha$ (se abrevia Tan $\alpha$ ) está dada pela razão $\frac{\overline{P C}}{\overline{A C}}$ entre o cateto oposto $\overline{P C}$ e o cateto adjacente $\overline{P C}$ do triângulo $A P C$. A diferença dos casos anteriores, nenhum dos segmentos tem uma medida constante e igual a um. Por isto, se faz necessária uma construção auxiliar que facilite a análise. Traçando uma reta tangente à circunferência que passe por $(\mathbf{1}, 0)$, esta intersecta os lados do ângulo nos pontos $B$ e $\boldsymbol{E}$ (ver Figura 4 c) formando-se assim o triângulo retângulo $O D A$ semelhante ao triângulo $O D B$. De maneira que, o estudo realizado neste último triângulo será igual se faz sobre o triângulo $O D A$. Considerando o triângulo $A E B$ tem-se que a $\operatorname{Tan} \alpha$ está definida pela razão $\frac{\overrightarrow{E B}}{\overline{A B}}$, onde $\overline{A B}=1$ é constante, o que significa que a Tan $\alpha$ fica referida pelo segmento $E B$. Ao igual que no Seno, se tomou a projeção ortogonal de dito segmento sobre o eixo y, o segmento dirigido $\overrightarrow{A F}$. De maneira que o módulo e sentido de dito vetor de cor morada representam a Tan $\alpha$ (ver Figura 4c).

Signos das razões trigonométricas. dada a natureza dos vetores representativos das razões trigonométricas estudadas, estes têm dois sentidos possíveis, para acima ou para baixo para o caso do Seno e a Tangente, e para a direita ou para a esquerda no Cosseno. Considera-se que uma razão é "positiva" se e somente se o sentido do vetor que a representa está para cima ou para a direita, e "negativa" no caso que o sentido de dito vetor esteja para abaixo ou para a esquerda. Com respeito ao módulo do vetor, este coincide com o valor absoluto da razão que represente. Por exemplo, no caso da Tangente, quando $\alpha=45^{\circ}$ o módulo do vetor é 1 e o sentido do vetor é para acima, por tanto se diz que a Tan $45^{\circ}$ é +1 ou simplesmente 1 . Em mudança, quando $\alpha=135^{\circ}$, o módulo do vetor que representa Tan $135^{\circ}$ ao também é 1 mas seu signo é negativo, já que o sentido de dito vetor está para a abaixo. Portanto, diz-se que a $\operatorname{Tan} 135^{\circ}=-1$.

\section{Considerações instrumentais}

Para a representação dos signos das razões trigonométricas no GeoGebra, considerou se o uso de um deslizador e três botões. O deslizador é uma ferramenta que permite representar um conjunto de valores, incluindo medidas angulares. Com isso é possível fazer variar a amplitude do ângulo $\alpha$ em tempo real. $\bigcirc$ referido deslizador pode realizar ajustes para que o ângulo varie em um intervalo de valores convenientes para o estudo. Por exemplo, aqueles que situam ao lado do ângulo em um ou outro quadrante do plano cartesiano Em alguns casos será conveniente usar opção "animação automática" sobre o deslizador, para ter uma visualização dinâmica das mudanças do valor do ângulo $\alpha$ e aqueles relacionados com o vetor que represente à cada razão (ver Figura 5a).

Os botões são ferramentas do GeoGebra que têm uma diversidade de aplicações no software, as quais dependerão da utilidade que Ihe dê o usuário. Para nosso caso, utilizouse para mostrar e ocultar alguns dos objetos representados na interface gráfica do programa, segundo considerou-se apropriado. No recurso, usam-se três botões para mostrar ou ocultar os vetores representativos das razões trigonométricas estudadas. Cabe destacar o uso de um texto dinâmico que mostra o valor absoluto da razão, que coincide com o módulo do vetor e a modificação da espessura, e cor dos vetores para uma melhor apreciação dos efeitos vinculados com o sentido dos vetores (ver Figura 5b). 


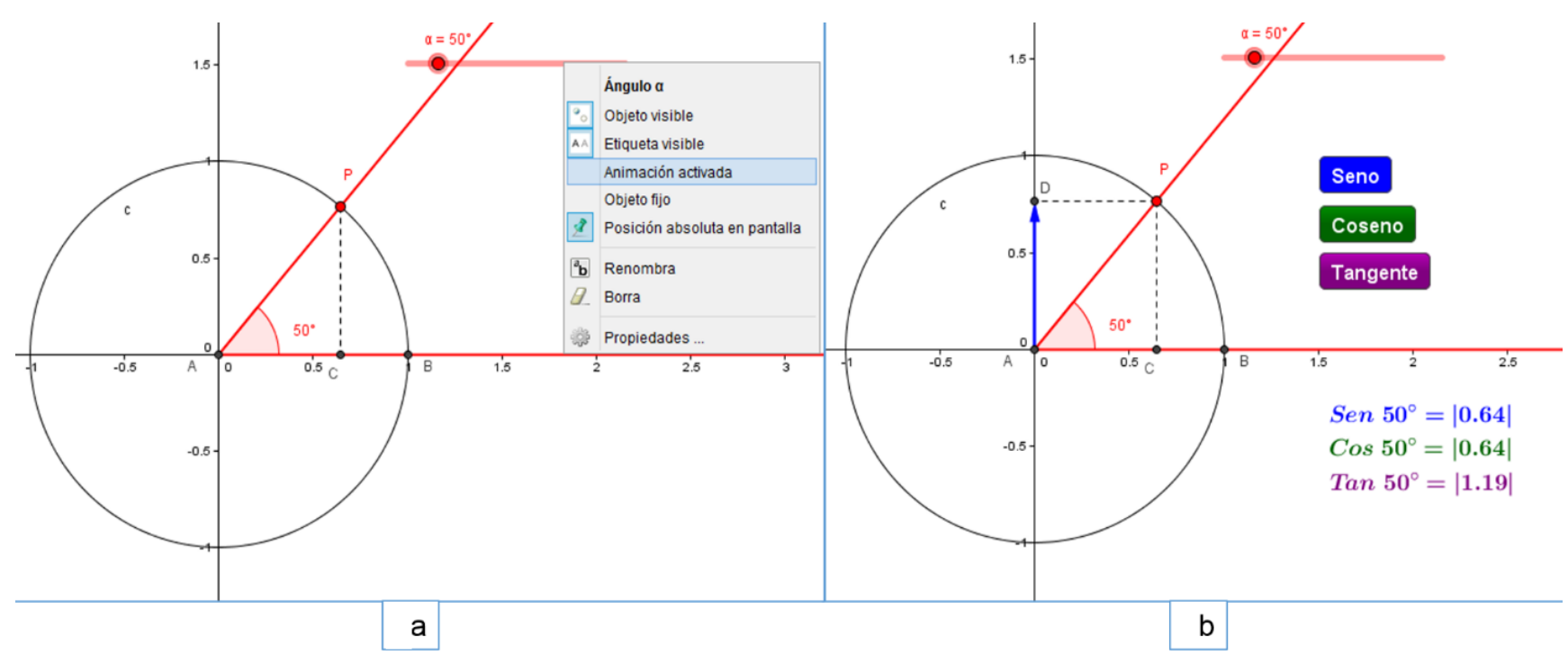

Figura 5. Deslizador e Botões

\section{Considerações didáticas}

Dado que a construção da circunferência unitária realiza-se sobre o sistema de coordenadas cartesianas, sugere-se realizar a análise para a cada quadrante do plano cartesiano por separado, ajustando o deslizador em intervalos de amplitudes angulares associadas à cada quadrante. De maneira, é possível também fazer o uso dos botões para visualizar o comportamento dos vetores referidos às razões Seno, Cosseno e Tangente separadamente. Por exemplo, ao ajustar o deslizar e selecionar o botão "Cosseno", determina-se o conjunto de ângulos para o segundo quadrante e observa-se o comportamento do vetor representativo do Cosseno neste quadrante. $O$ desenvolvimento deste processo no GeoGebra permite apreciar: (i) o sentido e módulo do vetor associado à cada razão trigonométrica por separado, (ii) a relação que existe entre estes e seu signo e valor, e (iii) o uso dos quadrantes cartesianos como referente para o estudo dos signos das razões trigonométricas.

\section{Sequência de análise}

A sequência que se indica a seguir, seguindo os parâmetros das considerações didáticas, consta de quatro etapas. A cada uma está referida aos quadrantes do plano cartesiano:

\section{Primeira Etapa: Seno, Cosseno e Tangente $0^{\circ} \leq \alpha \leq 90^{\circ}$}

Da análise do comportamento dos vetores para estes valores angulares no primeiro quadrante requer do ajuste conveniente dos valores mínimo e máximo do deslizador em $0^{\circ}$ e 90\%, respectivamente. Posteriormente, ativa-se a opção "animação automática" ao deslizador para observar que, os vetores representativos do $\operatorname{Sen} \alpha$ e a $\operatorname{Tan} \alpha$ em sentido para acima e o sócio ao $\operatorname{Cos} \alpha$ em sentido para a direita, se concluindo que, estas três razões são positivas para o primeiro quadrante (ver Figura 6). 


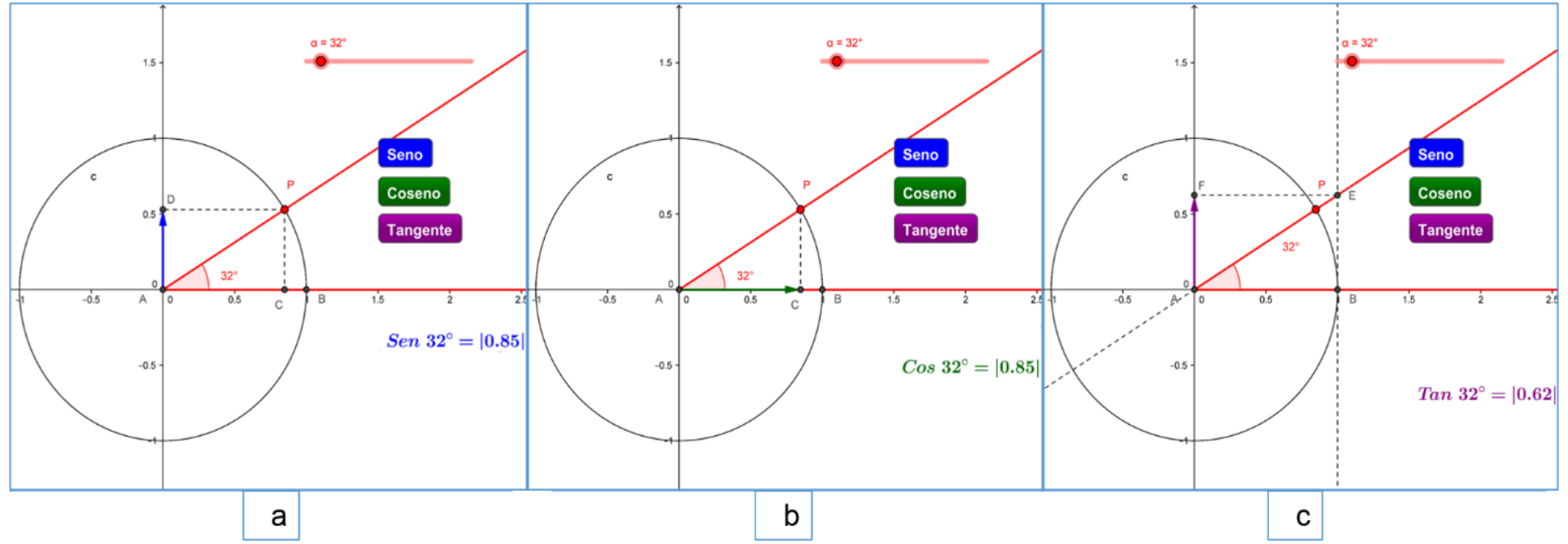

Figura 6. Seno, Cosseno e Tangente no quadrante I

Outras conjecturas que surgem depois da análise neste intervalo, tomando agora em conta o módulo da cada vetor, são aquelas referidas aos valores mínimos e máximos do primeiro quadrante:

Quando $\alpha=0^{\circ}$, Sen $\alpha=0, \operatorname{Cos} \alpha=1$ e $\operatorname{Tan} \alpha=0$

Quando $\alpha=90^{\circ}, \operatorname{Sen} \alpha=1, \operatorname{Cos} \alpha=0$ e $\operatorname{Tan} \alpha=+\infty$ (indeterminado). Com respeito à Tangente é importante observar que o vetor representativo deste desaparece no gráfico, quando o lado do ângulo em movimento é paralelo a reta tangente à circunferência em $(0,1)$.

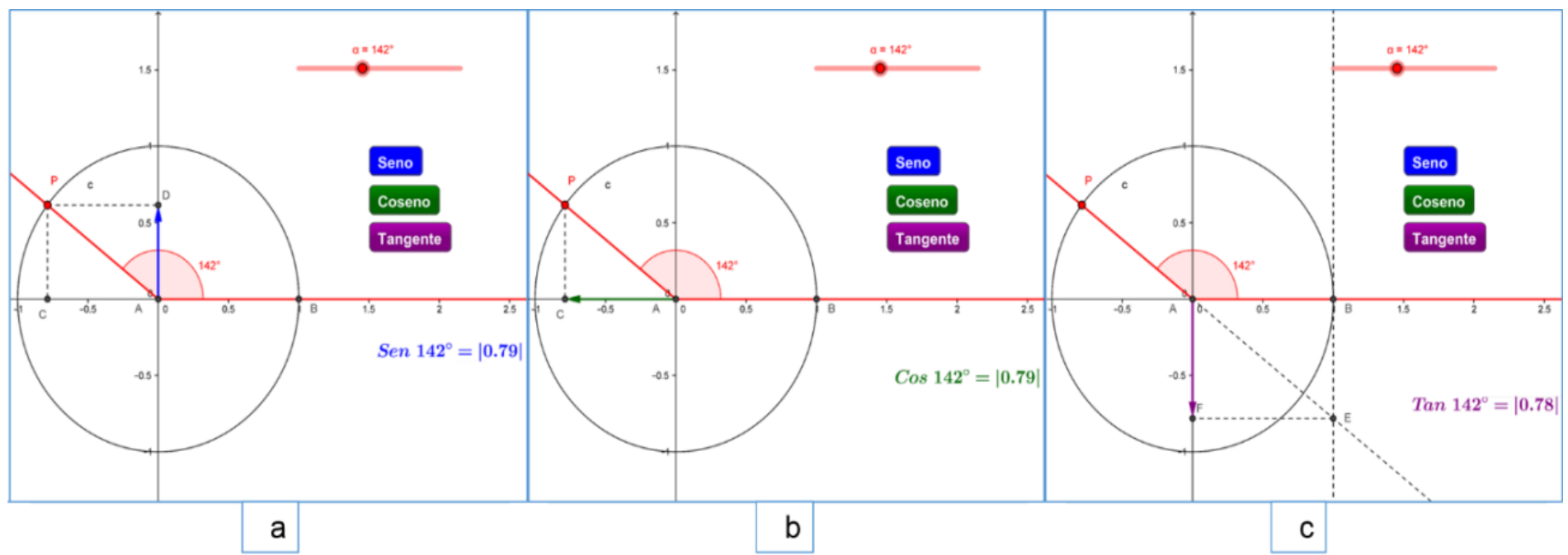

Figura 7. Seno, Cosseno e Tangente no quadrante II

Segunda Etapa: Seno, Cosseno e Tangente de $90^{\circ} \leq \alpha \leq 180^{\circ}$

No segundo quadrante, é conveniente ajustar os valores mínimos e máximos do deslizador em $90^{\circ}$ e $180^{\circ}$. Por meio da "animação automática" pode ser percebido que o vetor referente ao Seno segue estando em sentido para cima, igual ao primeiro quadrante, mas o vetor representativo da Tangente está em sentido para baixo e o que se refere ao Cosseno está em sentido para a esquerda, portanto se conjectura que o Seno é positivo e 
que o Cosseno e a Tangente são negativos para o intervalo $90^{\circ} \leq \alpha \leq 180^{\circ}$ associado ao segundo quadrante (ver Figura 7).

Nesta segunda etapa, o valor mínimo deste intervalo é $\alpha=90^{\circ} \mathrm{e}$ as possíveis conclusões para dito valor de $\alpha$ mostraram-se na etapa anterior. Com respeito ao valor máximo do intervalo para o segundo quadrante infere-se o seguinte:

Quando $\alpha=180^{\circ}, \operatorname{Sen} \alpha=0, \operatorname{Cos} \alpha=-1$ e $\operatorname{Tan} \alpha=0$.

\section{Terceira Etapa: Seno, Cosseno e Tangente de $180^{\circ} \leq \alpha \leq 270^{\circ}$}

Para esta análise, os valores do terceiro quadrante oscilam entre $180^{\circ}$ e $270^{\circ}$, portanto devem ser ajustado os valores mínimo e máximo do deslizador nestes valores ativando a "animação automática" no deslizador é possível ver que o sentido do vetor referido ao Seno está para abaixo, o que representa à Tangente está para acima e o do Cosseno está em sentido para a esquerda, por isso se conclui que, o Seno e Cosseno de $\alpha$ são negativos e a Tangente de $\alpha$ é positiva neste quadrante (ver Figura 8).

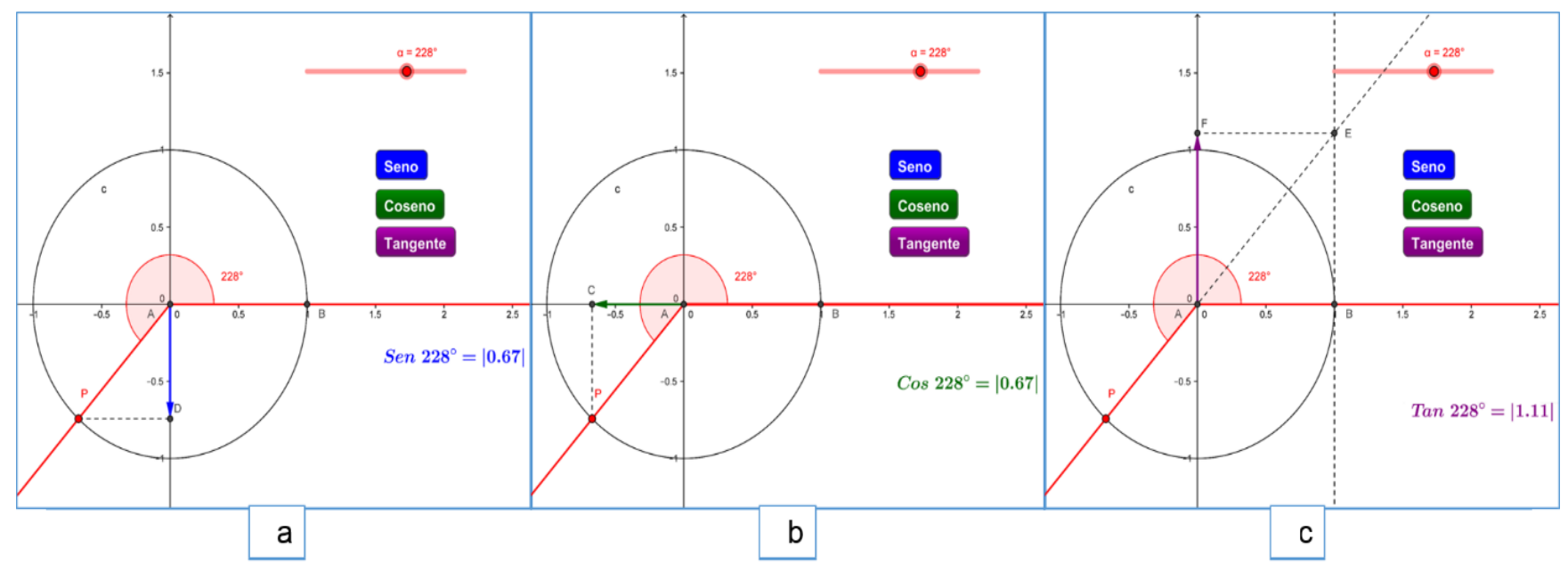

Figura 8. Seno, Cosseno e Tangente no III quadrante

As conjecturas sobre $\boldsymbol{\alpha}=\mathbf{1 8 0}^{\circ}$, que é o valor mínimo no intervalo do terceiro quadrante, se realizaram na segunda etapa da análise. Para o caso do valor máximo deste intervalo, $\boldsymbol{\alpha}=\mathbf{2 7 0}^{\circ}$ conclui-se o seguinte:

Sen $\boldsymbol{\alpha}=-1, \operatorname{Cos} \boldsymbol{\alpha}=\mathbf{0}$ e $\operatorname{Tan} \boldsymbol{\alpha}=+\infty$ (indeterminado). Para a Tangente pode ser percebido que sucede a mesma situação que para o caso de $\boldsymbol{\alpha}=\mathbf{9 0}^{\circ}$, o vetor representativo desta desaparece no gráfico, quando o lado do ângulo em movimento é paralelo à reta tangente à circunferência em $(\mathbf{0}, \mathbf{1})$.

Quarta Etapa: Seno, Cosseno e Tangente de $\mathbf{2 7 0}^{\circ} \leq \boldsymbol{\alpha} \leq \mathbf{3 6 0}^{\circ}$

Em esta última etapa, os valores de para o quarto quadrante são entre $270^{\circ}$ e $360^{\circ}$. Analogamente aos casos anteriores, devem ser ajustado os valores mínimos e máximos do deslizador em $270^{\circ}$ e $360^{\circ}$. Depois da variação de em este intervalo com \&a \#8220; Animação Automática", pode ser observado que os vetores representativos das raciocine Seno e Tangente estão em sentido para abaixo e o referido ao Cosseno está em sentido 
para a direita, portanto diz-se que, o Seno e Tangente são negativos e o Cosseno é positivo, para os valores de $\boldsymbol{\alpha}$ em o quarto quadrante (ver Figura 9).

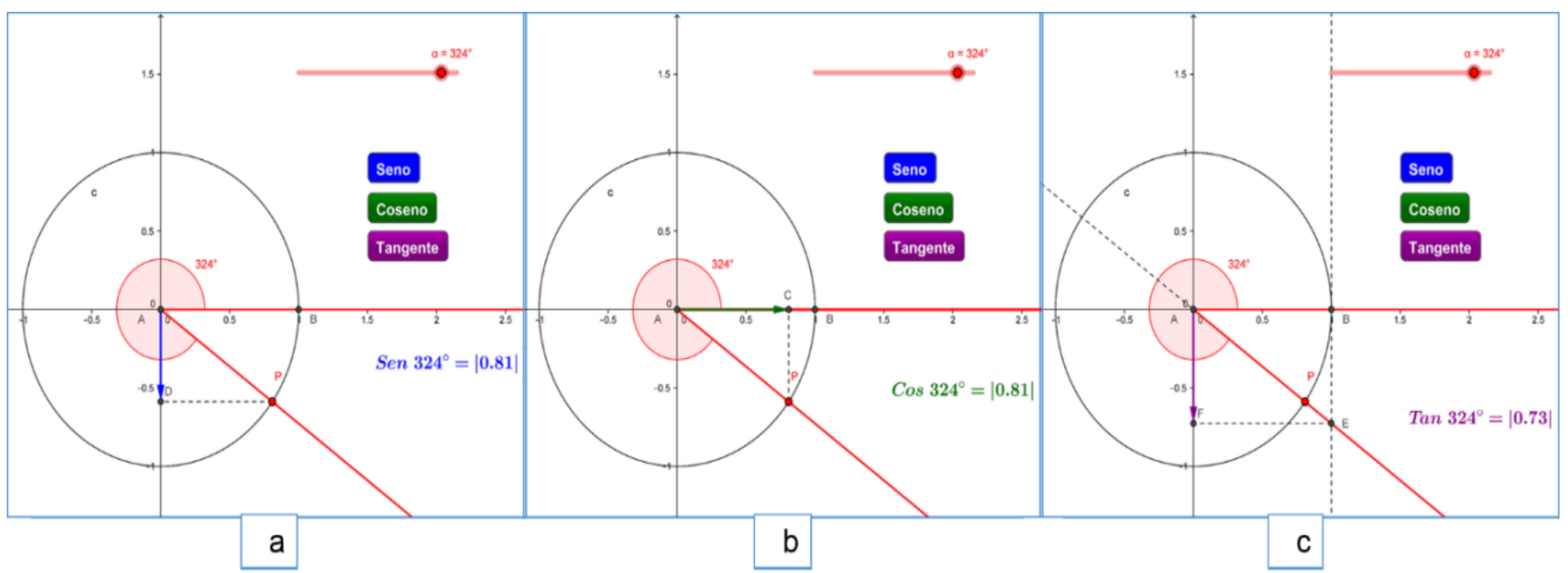

Figura 9. Seno, Cosseno e Tangente no quadrante IV

O valor mínimo no intervalo desta última etapa é de $\boldsymbol{\alpha}=\mathbf{2 7 0}^{\circ}$ e as conjecturas para o valor de $\boldsymbol{\alpha}$ apresentaram-se na etapa anterior. Para $\boldsymbol{\alpha}=\mathbf{3 6 0}^{\circ}$ que é o valor máximo do intervalo do quarto quadrante se deduze o seguinte:

\section{$\operatorname{Sen} \alpha=0, \operatorname{Cos} \alpha=1$ e $\operatorname{Tan} \alpha=0$.}

\section{Conclusões}

Ao longo deste trabalho apresentou-se o desenho e uma sequência de análise sobre um recurso que permite dotar de sentidos aos signos das razões trigonométricas: Seno, Cosseno e Tangente, sob o contexto de uma circunferência unitária construída com o software GeoGebra. A análise aqui descrita teve a intenção ajudar ao leitor a realizar suas próprias conjecturas sobre a interpretação geométrica dos vetores associados às respectivas razões, para compreender e confirmar, de forma visual, os resultados que apresentam as regras mnemotécnicas. Entre alguns resultados obtidos depois da construção desta circunferência no GeoGebra pôde ser estabelecido a relação entre os signos das razões trigonométricas com o sentido dos vetores representativos e, o valor absoluto da cada razão representada como o módulo do vetor correspondente, de maneira a permitir a determinação de certas características próprias das razões Seno, Cosseno e Tangente.

Por exemplo, conseguiu-se identificar os valores do ângulo para os quais a Tangente de certo ângulo é indeterminada e justificar a razão pela qual isto ocorre, fato que não pode ser explicado somente com o uso das regras mnemotécnicas. Estas e outras conjecturas que foram discutidas neste trabalho se devem aos atributos do GeoGebra postos em jogo no desenho do recurso e à sequência de análise proposta para o utilizar.

Acreditamos que através de sua manipulação os usuários possam compreender, analisar e tirar suas próprias conclusões a respeito ao que está sendo observado na tela do computador, que não é mais que os comportamentos geométricos dos vetores que representam às razões trigonométricas dantes mencionadas. 
Com o exposto, deseja-se mostrar: (i) formas de interatuar com um recurso tecnológico para dotar de sentido a certos conteúdos matemáticos que demandam de alguma maneira interpretação geométrica para seu adequado entendimento, pois estes costumam representar para o professor um grau de complexidade ao momento de realizar seu estudo em um meio estático, o qual tem gerado que eles abordem estes conteúdos com expediente didático meramente memorísticos e mecânicos, e (ii) ao reitera que o professores podem contar com estas novas alternativas didáticas como ferramentas que complementem sua prática e assim poder cumprir com as expectativas de aprendizagem que se propõem na Educação Matemática.

\section{Referências}

BORBA, M. D. C. Students' understanding of transformations of functions using multirepresentational software. 1993 Doctoral Dissertation - Cornell University Ithaca, NY. 1993

BORBA, M. D. C. \& VILLARREAL, M. Humans-with-media and the reorganization of mathematical thinking: information and communication technologies, modeling, experimentation and visualization. 1 ed. New York: Springer, 2005

BRITO, A. \& MOREY, B. Trigonometria: dificuldades dos professores de matemática do ensino fundamental. Revista Horizontes, v.22, p. 65-70, 2004

CHACÓN, A., SÁNCHEZ, A. \& QUIRÓS, C. Comprensión de las razones trigonométricas: niveles de comprensión, indicadores y tareas para su análisis. Revista Electrónica Actualidades Investigativas en Educación, v. 7 (2), p. 01-31, 2007

COUTINHO, Clara Pereira. TPACK: Em Busca de um Referencial Teórico para a Formação de Professores em Tecnologia Educativa. Revista Paidéi@. UNIMES VIRTUAL, Vol.2, Número 4, JUL. 2011. Disponível em http://revistapaideia.unimesvirtual.com.br.

DÍAZ, S. \& PIETRO, J. El análisis de los signos de las razones trigonométricas con tecnologías. Una manera de trascender las reglas prácticas. Comunicación presentada en el VIII Congreso Venezolano de Educación Matemática. Coro, 2013

DIKOVIĆ, L. Applications geogebra into teaching some topics of mathematics at the college level. Computer Science and Information Systems, v. 6 (2), p. 191-203, 2009

DUVAL, R. Quel cognitif retenir en didactique des mathématiques. Recherches en Didactique des Mathématiques, v. 16 (3), p. 349-382, 1996

FI, C.D. Preservice secondary school mathematics teachers' knowledge of trigonometry: cofunctions. Proceedings of the 28th annual meeting of the North American Chapter of the International Group for the Psychology of Mathematics Education, v. 2, p. 833-834, 2006

FIALLO, J. Estudio del proceso de Demostración en el aprendizaje de las Razones Trigonométricas en un ambiente de Geometría Dinámica. 2010 Tesis Doctoral Universitat de València, Valencia, 2010 
FIALLO LEAL, J.E., GUTIÉRREZ RODRÍGUEZ, A. Unidad de enseñanza de las razones trigonométricas en un ambiente Cabri para el desarrollo de las habilidades de demostración. Comunicación presentada en X Simposio de la SEIEM. Huesca, 2007

GUTIÉRREZ, R. \& PRIETO, J.L. Deformación y reflexión de funciones con GeoGebra. El caso de las parábolas definidas por la expresión $g(x)=a x^{2}$. Números, v. 88, p. 115-126, 2015

HOHENWARTER, M. Dynamic investigation of functions using geogebra. Proceedings of Dresden International Symposium on Technology and its Integration into Mathematics Education 2006. Dresden, Germany: DES-TIME. Disponível em: $<$ http://archive.geogebra.org/static/publications/2006-DES-TIME.pdf> Acesso em: 01/02/2015.

LABORDE, C., KYNIGOS, C., HOLLEBRANDS, K. \& STASSER, R. Teaching and learning geometry with technology. En A. Gutiérrez \& P. Boero (Eds.), Handbook of Research on the Psychology of Mathematics Education, pp. 275-304, 2006

LU, Y. W. A. Linking geometry and algebra: a multiple-case study of uppersecondary mathematics teachers' conceptions and practices of GeoGebra in England and Taiwan. 2008 Unpublished Master's thesis - University of Cambridge, Cambridge, 2008

MISHRA, P., \& KOEHLER, M. Technological pedagogical content knowledge: A framework for teacher knowledge. The Teachers College Record, v. 108 (6), p. 1017-1054, 2006

NAVARRO, E. Curso Proedeutico de Matemática. 1ed. Caracas: Litho-Tip, 1970 